\title{
Los estudios políticos en Perú: AUSENCIAS, DESCONEXIÓN DE LA REALIDAD Y LA NECESIDAD DE LA CIENCIA POLÍTICA COMO DISCIPLINA*
}

\author{
Martín Tanaka \\ Instituto de Estudios Peruanos, Perú
}

\begin{abstract}
Resumen
El siguiente trabajo presenta una crítica de los estudios políticos en Perú en las últimas décadas, y sostiene que el panorama está signado por la ausencia de trabajos serios sobre temas fundamentales, 0 por un abordaje basado en marcos conceptuales y teóricos inapropiados; esto es consecuencia de una excesiva politización, dentro de diversas corrientes de izquierda. Un hecho llamativo, porque si algo caracteriza la realidad política del Perú es precisamente su carácter poco previsible, indicativo de la autonomía de la política. En la actualidad se está en mejores condiciones para estudiar la política, desarrollo necesario porque casi todo está todavía por hacerse sobre temas políticos fundamentales.
\end{abstract}

Abstract

This essay presents a critical review of political science in Peru research over the last decades, and sustains that consequence of an excessive politization within different leftist currents, the landscape is marked by the absence of serious research on fundamental issues, and approaches based on inappropriate conceptual and theoretical frameworks. This is striking, because if peruvian political reality can be characterized by something, this would be its unpredictability, telling of the autonomy of politics. Currently, the discipline is in better conditions to analyze politics, a necessary development given that almost everything is still to be done on fundamental political issues.

\section{PALABRAS CLAVE • Ciencia Política • Perú • Politización • Desarrollo Disciplinario}

Este artículo es una revisión crítica de los estudios políticos en Perú en las últimas décadas, y sostiene que el panorama general está signado por una notoria ausencia de trabajos serios sobre temas fundamentales, o por un abordaje basado en marcos conceptuales y teóricos claramente inapropiados, desconectados y hasta a contracorriente de la naturaleza de las realidades políticas que se analizaban. También demuestra que esto es consecuencia de una excesiva politización, dentro de diversas corrientes de izquierda, en la que los estudiosos se pensaban más como ideólogos que como académicos, y que trajo también consigo un peso desmedido de corrientes de análisis sociologistas y estructuralistas, que menospreciaron el estudio de la política como campo autónomo de análisis. Esta desatención de la política es realmente llamativa, porque si algo caracteriza la realidad política del Perú de las últimas décadas es precisamente su carácter imprevisible, indicativo de la autonomía de la política frente a las determinaciones de las estructuras. En los últimos años, afortunadamente, se está en mejores condiciones para estudiar la política en Perú: la politización de la academia ha disminuido considerablemen-

* Esta es una primera versión de ideas en proceso de elaboración. Toda clase de comentarios son bienvenidos. 
te, se ha legitimado el estudio de la política desde cánones científicos, los politólogos, aunque todavía muy pocos, tienen mejor formación y están más al tanto de la literatura internacional, por lo que cabe abrigar moderadas esperanzas respecto al desarrollo de la Ciencia Política como disciplina. Desarrollo más que necesario, porque en términos de la producción de conocimiento sobre temas políticos fundamentales, casi todo está todavía por hacerse.

El recuento comienza desde la década de los años 70, cuando encontramos en las universidades departamentos de ciencias sociales relativamente consolidados. Hasta ese momento, el estudio de la política estaba, de un lado, en manos de abogados constitucionalistas, dentro de facultades de derecho y ciencias políticas; los abogados solían (suelen todavía) abordar la política dentro de los marcos del "viejo institucionalismo"1 (en el mejor de los casos), claramente insuficientes, al punto que prácticamente no hay ninguna producción relevante que reseñar desde esta perspectiva. De otro lado, están los trabajos sobre temas políticos realizados por historiadores, antropólogos y sociólogos, fuertemente marcados todos por una formación marxista-estructuralista ${ }^{2}$. Esto significa que la política aparecía subsumida dentro del análisis de las estructuras económicas y sociales, de las trayectorias y condiciones históricas, y más específicamente, de los intereses de las clases sociales. Las claves de la política se encontraban pues en la "anatomía de la sociedad civil", como diría Marx, entendida ésta en clave hegeliana. Ha sido señalado reiteradamente el hecho que Marx no desarrolló propiamente una teoría de la política y, en los años 60 y 70, ella terminó asumiendo la forma del estructuralismo francés (Althusser, Poulantzas) 3 .

Es muy llamativo que las cosas fueran así, porque precisamente entre 1968 y 1975 en el Perú teníamos un caso fascinante que ilustraba la autonomía de la política por encima de las determinaciones económicas y sociales, con el "Gobierno Revolucionario de la Fuerza Armada", encabezado por el General J uan Velasco. Lo extraordinario es que las Fuerzas Armadas peruanas siguieron un patrón distinto a las del Cono Sur, y al que habían seguido tradicionalmente; en vez de irrumpir en defensa del orden oligárquico, intervinieron con un ambicioso programa reformista, de una radicalidad nunca antes vista, que liquidó el orden tradicional. Esto, obviamente, no podía ser entendido desde aproximaciones que consideraban a las Fuerzas Armadas como parte de los aparatos de dominación de clase en el Estado. Sin embargo, la mayoría de los análisis de esos años enfatizaba, por el contrario, los "límites del reformismo", señalando que "en última instancia" los cambios eran "funcionales" al mantenimiento del capitalismo ${ }^{4}$. Es fuera del Perú que se encuentran aproximaciones más provechosas al examen de la dimensión política del velasquismo. Están, por ejemplo, los trabajos de Stepan (1978) y Trimberger (1977), que luego fueron recogidos por Skocpol (1985), y que dieron lugar a las nuevas corrientes institucionalistas que "traían de vuelta" el estudio del Estado. En esas investigaciones, el Perú aparecía al lado de Brasil, y al lado de Egipto, Turquía y J apón, como casos en los que se implementaban revoluciones "desde arriba", ilustrativas de la autonomía política de las instituciones estatales, por encima de la configuración del poder de las clases sociales.

Ver March y Olsen (1987).

En esos años existieron politólogos, ciertamente, pero muy pocos y sin capacidad de defender su campo disciplinario frente a las modas intelectuales y políticas imperantes. Así, en los primeros años de la facultad de ciencias sociales de la Universidad Católica existió una especialidad de ciencia política, pero duró apenas entre 1964 y 1971, y cerró por falta de alumnos (Tuesta, 2004). En esa facultad, la abrumadora mayoría de estudiantes optó por la carrera de sociología.

3 Pese a la gran influencia del marxismo, no hubo grandes desarrollos en cuanto a teoría marxista; dos excepciones muy creativas dignas de mención son Rochabrún (1976 y 1978); y Franco (1981).

$4 \quad$ Ver por ejemplo Lauer (1977). 
Hay algunos libros y textos que son hitos importantes que se deben resaltar puesto que son pertinentes para los temas que estamos discutiendo. Están, en primer término, aquellos escritos de Henry Pease sobre el gobierno militar (1979 y 1981). Son libros pioneros en un sentido politológico, porque analizan "la escena oficial", esto es, los conflictos entre los diversos grupos de poder al interior de la cúpula de las Fuerzas Armadas, rescatando la importancia y autonomía de este espacio; las críticas que recibió precisamente cuestionaban la falta de atención a las clases sociales, sus intereses y vínculos con esa escena oficial5. Sin embargo, Pease no se ubicaba en la línea de análisis de Stepan, Trimberger y Skocpol; por el contrario, al leer sus referencias teóricas, se alude a Poulantzas, a su conceptualización del Estado como campo de batalla por la hegemonía entre las clases, y al elusivo concepto de la "autonomía relativa" de la esfera institucional. El marco teórico de Pease era adecuado para estudiar la etapa del dominio oligárquico y los intentos democratizadores fallidos como los que expresó el APRA entre la década de los años treinta y sesenta, pero era totalmente inapropiado para el estudio de las reformas del gobierno militar. Los libros de Pease son por eso una clara muestra de una suerte de buena intuición politológica, pero que no pudo desarrollarse bien por las limitaciones de los discursos y teorías estructuralistas entonces en boga.

Otra referencia bibliográfica importante es la de McClintock y Lowenthal (1989), quienes compilaron en un libro ponencias presentadas en un seminario realizado a finales de los años 70 sobre el velasquismo ${ }^{6}$. Este volumen acaso es el más valioso de los que hay sobre el tema, y lo interesante es que en éste participan autores peruanistas de la academia estadounidense y algunos autores peruanos. Este tipo de esfuerzos combinados "norte-sur", son a mi juicio muy productivos, porque permiten combinar visiones más sólidas en términos teóricos con un conocimiento más profundo y en detalle de la realidad empírica? ${ }^{7}$.

Uno de los pocos autores peruanos que desde esos años aparecía integrado a los circuitos académicos internacionales de discusión es J ulio Cotler. Cotler participó no sólo en el seminario que dio origen al libro de McClintock y Lowentahl, también participó en el seminario de discusión sobre el "nuevo autoritarismo" en América Latina, en el que se debatieron las ideas de Guillermo O'Donnell sobre los Estados "burocrático-autoritarios". En esa oportunidad, Cotler (1979) presentó una interesante reflexión sobre las "sociedades de enclave", y sus diferencias con los Estados que ya habían cumplido con la "primera fase" del proceso de sustitución de importaciones. Esa discusión llevaba a una reflexión comparada del velasquismo peruano con otras experiencias similares, como las de los gobiernos de Rodríguez Lara en Ecuador o Torrijos en Panamá por los mismos años, pero esa línea de exploración no llegó a darse ni por Cotler ni por otros autores.

Luego vino la caída de Velasco en 1975, la "segunda fase" del gobierno militar, y la transición a la democracia, sucesos que se enmarcaban dentro de la "tercera ola democratizadora" (Huntington) que recorrió toda la región. La crisis de las dictaduras militares implicó la activación de un amplio

5 El trabajo de Pease descansó en las útiles cronologías políticas que editaba DESCO, que cubrieron los años 19681980. Afortunadamente, DESCO continuó con un banco de datos de noticias desde 1980 hasta la actualidad, aunque con algunas lagunas importantes.

La edición en inglés de este libro es de 1983; ver McClintock (1983).

En Franco (1983), encontramos un conjunto valioso de trabajos sobre diversos aspectos del velasquismo, entre ellos los políticos; sin embargo, es una compilación de trabajos sin norte teórico o analítico claro, más allá del intento de hacer un balance de las reformas. 
conjunto de movimientos de protesta en todos nuestros países, lo que llevó a los científicos sociales de toda la región a estudiar los "nuevos movimientos sociales", fuertemente inspirados por el pensamiento de Alain Touraine ${ }^{8}$ y bajo el importante estímulo institucional del Consejo Latinoamericano de Ciencias Sociales (CLACSO), en investigaciones que se desarrollaron sobre todo en la primera mitad de la década de los años $80^{\circ}$. La agenda de los nuevos movimientos sociales tuvo así un fuerte énfasis sociológico ${ }^{10}$. Lo interesante es que, paralelamente, un equipo convocado por el Wilson Center en Washington, D.C., iniciaba por los mismos años otro ambicioso programa de investigaciones sobre las transiciones a la democracia, pero con un énfasis politológico, podríamos decir, al privilegiar en el análisis de las transiciones a las interacciones entre las élites políticas y sociales ${ }^{11}$. El desenlace de esta historia de paradigmas en competencia lo conocemos: mientras la literatura de las transiciones se reveló muy provechosa e influyente, la de los nuevos movimientos sociales progresivamente declinó; es que las expectativas puestas en los actores que serían "fundadores de un nuevo orden" (Ballón, 1986a) no se cumplieron; por el contrario, poco a poco, en todos nuestros países, los movimientos se desmovilizaron, una vez que los partidos políticos empezaron a ocupar el papel que les corresponde en una democracia representativa. Nuevamente encontramos un desencuentro entre las preocupaciones implícitas en la aproximación a los fenómenos políticos en las ciencias sociales peruanas (y latinoamericanas en este caso) y lo que ocurría en la realidad política.

En el Perú, esta temática puede ilustrarse contrastando los textos publicados por Ballón (1986 y 1986a) y el capítulo de Cotler (1988a) en el volumen de O'Donnell et al. (1988) sobre las transiciones. Ballón editó un conjunto de trabajos que compartían la expectativa del potencial de cambio de diversos movimientos sociales, y una llamativa ausencia en el análisis de la dimensión política. La política era todavía percibida como una suerte de epifenómeno, que no merecía una atención en sí misma ${ }^{12}$. De otro lado, está Cotler, quien participó del proyecto sobre transiciones del Wilson Center, y escribió el capítulo sobre Perú, desde una perspectiva que recoge el enfoque linziano del proyecto, y lo combina con un fuerte peso de sociología histórica, aproximación que ya había esbozado en su libro Clases, Estado y nación en el Perú (1978) ${ }^{13}$.

Si se analiza la apasionante década de los años 80 , signada por una gran riqueza de acontecimientos políticos, y consideramos los estudios políticos publicados hasta los primeros años de la década de los 90, encontramos muy pocos estudios politológicos propiamente dichos que resal-

3 Es muy curioso cómo López (1992 y 2001) aplica el modelo teórico de las transiciones, pero de una manera extemporánea,

para analizar distintas etapas del fujimorismo.

Ver Touraine (1977), entre muchos otros.

Ver por ejemplo J elín et al. (1986).

Es importante recordar que se pudieron estudiar los movimientos sociales y su dinámica desde otras ópticas, que enfatizaran la acción de empresarios, políticos y diversos actores externos que asumen los costos de la acción colectiva, lo que permite estudiar la relación de los movimientos sociales con partidos y el Estado; es decir, frente al "paradigma de la identidad" tourainiano existía en los mismos años el paradigma de la "movilización de recursos", más cercano a la academia norteamericana. El peso de los enfoques tourainianos fue abrumador, no sólo en Perú, sino en toda la región, al punto que prácticamente no existen trabajos desde otros paradigmas teóricos. En Perú, recién es con Pásara, et al. (1991), que se propone pensar los movimientos sociales desde ópticas alternativas.

Ver O'Donnell y Schmitter (1988). En el Perú, el estudio de los actores políticos hasta esos años se circunscribía al debate al interior de los partidos de izquierda. Ver Letts (1981), y Nieto (1983).

Es interesante ver el ensayo de Ballón (1989), no publicado en el Perú, en el que se intenta un abordaje al estudio del sistema político peruano, pero desde una perspectiva de sociología política; es decir, no se llega a estudiar propiamente el sistema político. 
tar, que contrastan con la vitalidad de la producción sociológica, abocada fundamentalmente al estudio de los diversos actores sociales, especialmente dentro de los sectores populares ${ }^{14}$. Los estudios políticos son, por lo general, poco más que comentarios periodísticos o ensayos que adolecen de las virtudes y defectos del género: de un lado pueden ser sugerentes y brillantes, pero pecan también por especulativos, impresionistas, y politizados; el estudio de la política quedó subordinado en gran medida a las apuestas y la militancia política ${ }^{15}$. Precisamente cuando se estaba formando un sistema de partidos y una institucionalidad democrática, el énfasis de los estudios seguía todavía puesto en lo social y en los límites de la democracia ${ }^{16}$. Nuevamente, para encontrar estudios más profundos y sistemáticos de la realidad política peruana en esos años, hay que buscar fuera del país ${ }^{17}$.

El inicio de los 90, con la inesperada elección de Alberto Fujimori (1990), marca nuevamente una excelente muestra de la autonomía de la política frente a determinaciones estructurales. Cuando se esperaba una gran confrontación clasista y una alta polarización ideológica, lo que terminó ocurriendo fue un vacío de representación, la llegada al poder de un outsider, y el posterior colapso del sistema de partidos. Es interesante notar cómo escritos como los de Grompone y Degregori (1991) apelan a explicaciones de corte sociológico y cultural para dar cuenta de un fenómeno que llama la atención más bien por su especificidad política; es revelador contrastar esos trabajos con los de Schmidt (1996) y Cameron (1997), nuevamente académicos que producen desde los Estados Unidos, quienes sí llaman la atención sobre el peso de las reglas electorales y de las estrategias e interacciones de los actores para dar cuenta de ese desenlace.

El fujimorismo y su consolidación, en medio de condiciones sumamente adversas, nuevamente ilustran la autonomía de la política frente a otras esferas sociales; de hecho, si se puede encontrar una constante en la política peruana es su imprevisibilidad, sus giros inesperados y desenlaces sorprendentes ${ }^{18}$. Afortunadamente, des de la segunda mitad de la década de los años 90 empiezan a aparecer en Perú más trabajos que se ocupan de la dinámica política peruana. Varios elementos explican esto, siendo el principal el colapso de la izquierda ${ }^{19}$ y con ella su ideología y modelos teóricos, que permitieron tomar en serio la autonomía de la política y lograr una mayor

14 Una revisión bibliográfica de la amplia literatura sobre las relaciones entre sociedad y Estado en Perú puede verse en Tanaka (2002b). Algunos de los pocos estudios políticos importantes que se pueden citar de autores peruanos serían Grompone, 1991; López, 1991; Pásara y Parodi, eds., 1988; Pease, 1986 y 1988; y Campodónico et al., 1993, Castillo, 1992, Paredes y Sachs, 1991, y Webb, 1987 y 1988, desde una perspectiva de economía política.

15 Al respecto ver Gonzales, 1999, quien estudia a los intelectuales reunidos en la revista El Zorro de Abajo. En ella, autores como López divulgaron las lecturas que sobre Gramsci hicieron autores como Portantiero y Aricó desde la Argentina, e introdujeron al debate el tema de la lucha por la hegemonía al interior del Estado. Ver también López, 1991. Hablando de intelectuales y política, tal vez no sea casual que hacia finales de los años 80 se haya propiciado una interesante reflexión sobre el pensamiento político peruano: ver Adrianzén, ed., 1987 y 1990.

16 Ver por ejemplo Ballón et al. (1990).

17 Ver los textos de Scurrah (1986), Aggarwal y Cameron (1994), y Conaghan y Malloy (1994) sobre el segundo gobierno de Belaunde, y los de Bonilla y Drake, eds., (1989), Cameron (1994), Crabtree (1992) y Graham (1992), sobre el gobierno de García. El volumen de Bonilla y Drake es nuevamente un buen ejemplo de lo útil que resultan proyectos en colaboración "norte-sur". Cabe una especial mención al trabajo de Cotler (1995) sobre los partidos en Perú, en la valiosa compilación de Mainwaring y Scully. Nuevamente, Cotler aparece siendo parte de proyectos comparados fundamentales, pero nuevamente como una figura solitaria, en un volumen publicado por Stanford University Press, con escasa difusión en Perú.

18 Ver Tanaka (2005). Esto vale también para la inesperada caída del fujimorismo.

19 Sobre el tema ver Tanaka (2002) 
profesionalización de los estudios políticos, tomando en serio criterios académicos y científicos. El final del modelo del "intelectual comprometido" permitió además superar ciertos prejuicios y facilitó el diálogo con la ciencia política norteamericana y ang losajona en general, que precisamente reivindican el carácter de la ciencia política como disciplina de investigación empírica, distinta al ensayismo político, la sociología política, la filosofía política, el derecho constitucional, y otras disciplinas afines. Esto ha permitido la aparición y desarrollo de estudios propiamente politológicos, en diálogo con investigadores del extranjero, cosa también facilitada por las comunicaciones, internet, y otras herramientas.

Algunos hitos importantes en este sentido son los trabajos de Fernando Tuesta (1995) des de una línea institucionalista, centrada en el examen de los sistemas electorales ${ }^{20}$; están también las compilaciones del mismo autor (Tuesta, 1996 y 1999), en las que reúne las ponencias sobre temas políticos de dos de los congresos de la Latin American Studies Association, siguiendo con la tradición de intercambio "norte-sur" entre académicos peruanos y de los Estados Unidos. En la misma línea cabe destacar las ediciones de Cameron y Mauceri (1997) y Crabtree y Thomas (1998). En Perú también aparecerán posteriormente trabajos de autores peruanos desde perspectivas netamente politológicas ${ }^{21}$; afortunadamente, cada vez más aparecen trabajos sobre distintos aspectos de la realidad política del país que la abordan apropiadamente, respetando la autonomía de su campo temático.

En los últimos años se registra también un creciente interés por la política y su estudio en el país en general, lo que ha llevado a la creación de varias maestrías en ciencia política y gobierno en diversas universidades públicas y privadas 22 ; próximamente en este mismo año (2005) la Universidad Católica abrirá lo que podríamos considerar la primera licenciatura de ciencia política en Perú propiamente dicha ${ }^{23}$. También es importante mencionar que cada vez más hay estudiantes que buscan seguir postgrados en ciencia política en universidades del extranjero. Todo esto es fundamental para el desarrollo de la disciplina, porque hasta el momento la mayoría de profesores en programas de ciencia política no son politólogos, sino que provienen de otras áreas.

Esperemos que estos avances permitan en el futuro cubrir los enormes vacíos que todavía existen para alcanzar un conocimiento básico de la política en Perú. En realidad, casi todo está todavía por hacerse. No es exageración. Consideremos brevemente este asunto. Para empezar, casi toda la historia política del país está por escribirse desde un punto de vista politológico. Sin retroceder mucho, si se mira el siglo XX, se encuentra muy poco sobre los años de la llamada "República Aristocrática" (finales del siglo XIX hasta 1919) y el leguiísmo (1919-1930). No hay estudios profundos sobre cómo se hacía política en la época de la oligarquía, el funcionamiento del congreso, la realización de elecciones, las relaciones entre los notables de Lima con los operadores

20 Cabe mencionar que Tuesta ya había desde 1987 emprendido la necesaria tarea de recoger y ordenar resultados electorales en Perú político en cifras: élite política y elecciones (Lima Fundación Ebert), con ediciones en 1987, 1994 y 2001. Tuesta siguió el camino iniciado por Roncagliolo (1980).

21 Ver p.e, Tanaka, 1998; Lynch, 1999; Reyna, 2000, entre otros, sobre la democracia en Perú en la década de los años 80.

22 Cabe destacar la Maestría en Ciencia Política de la Universidad Católica, abierta en 1998, y la Mención en Estudios Políticos de la Maestría en Sociología, de la Universidad de San Marcos, que cobró impulso en esos mismos años.

23 Si bien hay otros programas de grado en Ciencia Política en otras universidades (como Federico Villarreal, por ejemplo), un examen de su currículo muestra que bajo ese nombre terminan haciéndose muy distintas cosas, diferentes a lo que convencionalmente conocemos como ciencia política. 
políticos en las provincias, y las relaciones entre éstos y los sectores populares en general, y la población indígena en particular ${ }^{24}$. No existen casi estudios sobre el periodo 1930-1968; para empezar, no existe una sola historia política del APRA que evite ya sea la descalificación a priori como la hagiografía; lo mismo puede decirse de la actuación del Partido Comunista y otros partidos durante ese periodo. No hay casi nada escrito sobre los experimentos democráticos restringidos ocurridos entre 1939 y 1948 y entre 1956 y $1968^{25}$. Todo este periodo, lamentablemente, no está estudiado, y esto es consecuencia de una concepción según la cual durante el orden oligárquico la política era la simple prolongación de los intereses de la clase dominante, y por lo tanto, no digna de atención. Sin embargo, debería pensarse el periodo como un intento de construcción de partidos y de un sistema de partidos que podría haber tenido éxito, de no mediar las intervenciones autoritarias ${ }^{26}$.

Como ya se señaló, hay todavía muy poco escrito sobre asuntos políticos fundamentales del gobierno militar; si bien hay una literatura significativa sobre el periodo de transición ${ }^{27}$, falta decir casi todo de los gobiernos de Belaúnde y García: los trabajos existentes hacen evaluaciones globales, pero no analizan temas específicos. En cuanto al periodo que va del fujimorismo hasta el momento actual hay muchos más estudios valiosos, pero todavía hay lagunas gigantescas respecto a todo el periodo que se inició en 1980: no contamos con estudios profundos sobre el congreso, el proceso legislativo, las relaciones entre los congresistas y sus representados, etc.; estudios que analicen la dinámica interna de los partidos; o estudios de economía política sobre las grandes medidas de política económica y social, y la intervención de poderes de facto ${ }^{28}$. Hay otras dos ausencias ominosas que quiero resaltar: una es la de los estudios políticos regionales, expresiva de una actividad académica casi exclusivamente centrada en Lima, en apenas un puñado de instituciones que en medio de grandes dificultades hacen todavía investigación (como el Instituto de Estudios Peruanos, DESCO y centros de investigación en universidades como la Católica, San Marcos y Pacífico ${ }^{29}$. Otra ausencia notoria es la de investigaciones que ubiquen al Perú en una perspectiva comparada, pero pensada desde el país ${ }^{30}$.

Todo esto en cuanto a estudios sobre temas fundamentales. En términos del desarrollo institucional de la ciencia política, hay desgraciadamente muy poco que decir. Como ya se mencionó, apenas en los últimos años es que se han abierto algunos programas universitarios en la disciplina, por lo que todavía no existen egresados propiamente dichos ni desarrollos profesionales y laborales que se puedan evaluar; si bien hay egresados de programas de maestría en ciencia política, sus referencias más importantes están asociadas a sus estudios de grado (derecho, sociología, principalmente), y el programa de grado en ciencia política de la Universidad Católica apenas empieza este año. No es de sorprender que no exista una sola revista de ciencia política o de estudios políticos en general que

\footnotetext{
Con la excepción de algunos estudios pioneros de Cotler y otros de autores como Pedro Planas y Cristóbal Aljovín.

Algunas excepciones son los libros de Portocarrero (1983) y Kuczynski (1980).

Ver al respecto el muy sugerente texto de Cotler (1988).

Cotler (1998a); Pease (1981); Sanborn (1991); Lynch (1992).

Con la excepción parcial de los trabajos de Francisco Durand sobre la élite empresarial y sus relaciones con la política; ver Durand (2003).

29 Ver al respecto Rénique (2004), para el caso de Puno.

30 Ciertamente hay muchísimos trabajos que ubican al Perú en perspectiva comparada, pero llevados a cabo por académicos de otros países. En el Perú no hay casi intentos similares, salvo en Tanaka (2006, 2003 y 2002a). No considero aquí trabajos desde la perspectiva de las relaciones internacionales, que creo ameritan un tratamiento diferente.
} 
esté a la altura de criterios académicos internacionales, y en general el desarrollo institucional de la disciplina enfrenta el límite que sufre el sistema universitario y de investigación científica del país en general, signado por la precariedad y la escasez de recursos. Hace falta también lograr mayor pluralismo ideológico y teórico en nuestra comunidad de investigadores en temas políticos, aún dominada por sentidos comunes de izquierda. Hace falta también lograr mayor presencia e influencia pública, desde una perspectiva profesional y no partidaria o ideológica, para contribuir en los debates nacionales e incidir en un mejor diseño de las políticas públicas. En general, hay todavía desconfianza respecto a los "analistas políticos", que son percibidos como defensores de posiciones predeterminadas por sus opciones políticas e ideológicas, aunque se ha avanzado mucho en el último tiempo en cuanto a ganar un espacio más "técnico" para los politólogos.

Como puede verse, en realidad, casi todo está todavía por hacerse. Lo bueno es que se está en mejores condiciones que antes, tanto intelectuales como institucionales, para emprender las tareas pendientes.

\section{REFERENCIAS}

Adrianzén, Alberto (ed.). 1990. Pensamiento político peruano, 1930-1968. Lima: DESCO.

Adrianzén, Alberto (ed.). 1987. Pensamiento político peruano. Lima: DESCO.

Aggarwal, Vinod y Maxwell Cameron. 1994. "Modelling Peruvian Debt Rescheduling in the 1980s". Studies in Comparative International Development 29 (2): 48-81

Ballón, Eduardo. 1989. "Estado, sociedad y sistema político peruano". En Los sistemas políticos en América Latina, editado por Lorenzo Meyer y J osé Luis Reyna. México D.F.: Siglo XXI.

Ballón, Eduardo (ed.). 1986. Movimientos sociales y crisis: el caso peruano. Lima: DESCO.

Ballón, Eduardo (ed.). 1986a. Movimientos sociales y democracia: la fundación de un nuevo orden. Lima: DESCO.

Ballón, Eduardo et.al. 1990. Movimientos sociales: elementos para una relectura. Lima: DESCO.

Bonilla, Heraclio y Paul Drake (eds.). 1989. EI APRA de la ideología a la praxis. Lima: Centro Latinoamericano de Historia Social, Center for Iberian and Latin American Studies, y Editorial y Productora Gráfica Nuevo Mundo.

Cameron, Maxwell. 1994. Democracy and authoritarianism in Peru. Political coalitions and social change. New York: St. Martin's Press.

Cameron, Max y Philip Mauceri (eds.). 1997. The Peruvian Labyrinth. University Park, PA: Pennsylvania State University Press. Campodónico, Humberto, Manuel Castillo y Andrés Quispe. 1993. De poder a poder. Grupos de poder, gremios empresariales y política macroeconómica. Lima: DESCO.

Castillo, Manuel. 1992. La escena astillada: crisis, acumulación y actores sociales. Lima, DESCO.

Conaghan, Catherine M. y James M. Malloy. 1994. Unsettling Statecraft. Democracy and Neoliberalism in the Central Andes. Pittsburgh: University of Pittsburgh Press.

Cotler, J ulio. 1995. "Political Parties and the Problems of Democratic Consolidation in Peru". En Building Democratic Institutions. Party Systems in Latin America, editado por Scott Mainwaring y Timothy Scully. California: Stanford University Press, 232-353.

Cotler, Julio. 1988. "Los partidos políticos y la democracia en el Perú". En Democracia, sociedad y gobierno en el Perú, editado por Luis Pásara y J orge Parodi. Lima: CEDYS, 151-192.

Cotler, Julio. 1988a. "Las intervenciones militares y la 'transferencia del poder a los civiles' en Perú". En Transiciones desde un gobierno autoritario Vol. 2: América Latina (1986), editado por O'Donnell, Guillermo, et al. Buenos Aires: Paidós, 225-259.

Cotler, Julio. 1979. "State and Regime: Comparative Notes on the Southern Cone and the Enclave Societies". En The New Authoritarianism in Latin America, editado por David Collier. Princeton: Princeton University Press, 255-282.

Cotler, Julio. 1978. Clases, Estado y Nación en el Perú. Lima: Instituto de Estudios Peruanos.

Crabtree, J ohn. 1992. Peru under García. An opportunity lost. Pittsburgh: University of Pittsburgh Press. 
Crabtree, J ohn y J im Thomas (eds.). 1998. Fujimori's Peru: The Political Economy. London: Institute of Latin American Studies, University of London.

Gonzales, Osmar. 1999. Señales sin respuesta. Los Zorros y el pensamiento socialista en el Perú, 1968-1989. Lima: PREAL. Graham, Carol. 1992. Peru's APRA. Parties, Politics, and the Elusive Quest for Democracy. Boulder: Lynne Rienner Publishers, Inc. Degregori, Carlos Iván y Romeo Grompone. 1991. Elecciones 1990: Demonios y redentores en el nuevo Perú. Una tragedia en dos vueltas. Lima: Instituto de Estudios Peruanos.

Durand, Francisco. 2003. Riqueza económica y pobreza política: reflexiones sobre las élites del poder en un país inestable. Lima: PUCP.

Franco, Carlos (coord.). 1983. El Perú de Velasco. Lima: CEDEP.

Grompone, Romeo. 1991. El velero en el viento. Política y sociedad en Lima. Lima: Instituto de Estudios Peruanos.

Jelín, Elizabeth et al. 1986. Los movimientos sociales ante la crisis. Buenos Aires: CLACSO.

Kuczynski, Pedro Pablo. 1980. Democracia bajo presión económica: el primer gobierno de Belaúnde, 1963-1968 (1977). Lima: Mosca Azul eds.

Lauer, Mirko (ed.). 1977. El reformismo burgués, 1968-1976. Lima: Mosca Azul.

Letts, Ricardo. 1981. La izquierda peruana. Organizaciones y tendencias. Lima: Mosca Azul.

Lynch, Nicolás. 1992. La transición conservadora. Movimientos sociales y democracia en el Perú, 1975-1978. Lima: El Zorro de Abajo.

López, Sinesio. 2001. “Perú, 2000-2001: la transición democrática y el gobierno de transición”. En Las tareas de la transición democrática, editado por Cecilia Anicama et al. Lima: Comisión Andina de Juristas, 59-90.

López, Silesio. 1992. "Perú: golpe, democracia y democradura". Cuestión de Estado 4/5: 28-35.

López, Silesio. 1991. El dios mortal: Estado, sociedad y política en el Perú del siglo XX. Lima: IDS.

March, James y Johan Olsen. 1987. "The New Institutionalism: Organizational Factors in Political Life". American Political Science Review 78 (septiembre): 734-749.

McClintock, Cynthia (ed.). 1983. The Peruvian Experiment Reconsidered. Princeton: Princeton University Press.

McClintock, Cynthia y Abraham F. Lowenthal (comp.). 1989. El gobierno militar: una experiencia peruana. Lima: Instituto de Estudios Peruanos.

Nieto, J orge. 1983. Izquierda y democracia en el Perú, 1975-1980. Lima: DESCO.

O'Donnel, Guillermo y Philip Schmitter. 1988. Transiciones desde un gobierno autoritario. Vol. 4: Conclusiones tentativas sobre democracias inciertas. Buenos Aires: Paidós.

Paredes, Carlos, y J effrey Sachs (eds.). 1991. Estabilización y crecimiento en el Perú. Lima: GRADE.

Pásara, Luis et al. 1991. La otra cara de la luna. Nuevos actores sociales en el Perú. Buenos Aires: CEDYS.

Pásara, Luis y Jorge Parodi (eds.). 1988. Democracia, sociedad y gobierno en el Perú. Lima: CEDYS.

Pease, Henry. 1988. Democracia y precariedad bajo el populismo aprista. Lima: DESCO.

Pease, Henry. 1986. "El segundo belaundismo en el nuevo régimen político peruano". Informe de Investigación. Mimeo, DESCO.

Pease, Henry. 1981. Los caminos del poder. Tres años en la escena política peruana (1975-1978). Lima: DESCO.

Pease, Henry. 1979. El ocaso del poder oligárquico. Lucha política en la escena oficial, 1968-1975. Lima: DESCO.

Portocarrero, Gonzalo. 1983. De Bustamante a Odría: el fracaso del Frente Democrático Nacional, 1945-1948. Lima: Mosca Azul.

Rénique, J osé Luis. 2004. La batalla por Puno. Conflicto agrario y nación en los Andes peruanos. Lima: Instituto de Estudios Peruanos - CEPES - SUR.

Reyna, Carlos. 2000. La anunciación de Fujimori: Alan García 1985-1990. Lima: DESCO.

Roncagliolo, Rafael. 1980. ¿Quién ganó? Elecciones 1931-80. Lima: DESCO.

Sanborn, Cynthia. 1991. The Democratic Left and the Persistance of Populism in Peru: 1975-1990. Tesis doctoral. Cambridge: The Department of Government, Harvard University.

Schmidt, Gregory. 1996. "Fujimori's 1990 upset victory in Peru: electoral rules, contingencies, and adaptative strategies". Comparative Politics 28 (3): 321-354.

Scurrah, Martin. 1986. The Latin American State and the Politics of Austerity: Peru, 1980-1985. Melbourne: La Trobe Institute of Latin American Studies. 
Skocpol, Theda. 1985. "Bringing the State Back In: Strategies of Analysis in Current Research". En Bringing the State Back In, editado por P. Evans, D. Rueschemeyer y T. Skocpol. Cambridge: Cambridge University Press, 3-37.

Stepan, Alfred. 1978. The State and Society: Peru in Comparative Perspective. Princeton, N.J .: Princeton University Press.

Tanaka, Martín. 2006. "From Crisis to Collapse of the Party Systems, and Dilemmas of Democratic Representation: the Cases of Peru and Venezuela". En The Crisis of Democratic Representation in the Andes, editado por Ana María Bejarano, Scott Mainwaring y Eduardo Pizarro. Stanford: Stanford University Press (en prensa).

Tanaka, Martín. 2005. "Peru 1980-2000: Chronicle of a Death Foretold? Determinism, Political Decisions and Open Outcomes". En The Third Wave of Democratization in Latin America. Advances and Setbacks since 1978, editado por Frances Hagopian y Scott Mainwaring. Cambridge: Cambridge University Press (en prensa).

Tanaka, Martín. 2003. "Los sistemas de partidos en los países andinos: autoritarismos competitivos y reformismo institucional". Ponencia presentada en la conferencia Crisis in the Andes: Managing Regional Order. Center for International Studies, University of Oxford.

Tanaka, Martín. 2002. "Ideologización y 'basismo' vs. pragmatismo y búsqueda del centro político. De cómo esa tensión dividió y liquidó a la izquierda en el Perú". Ponencia presentada en el seminario "La izquierda en América Latina", del Grupo de Trabajo de CLACSO sobre Partidos Políticos y Sistemas Electorales. Universidad de Campinas, octubre.

Tanaka, Martín. 2002a. La situación de la democracia en Colombia, Perú y Venezuela a inicios de siglo. Lima: Comisión Andina de Juristas.

Tanaka, Martín. 2002b. "Las relaciones entre Estado y sociedad en el Perú: desestructuración sin reestructuración. Un ensayo bibliográfico". América Latina Hoy 31 (agosto): 189-218.

Tanaka, Martín. 1998. Los espejismos de la democracia. El colapso del sistema de partidos en el Perú, 1980-1995, en perspectiva comparada. Lima: Instituto de Estudios Peruanos, 280.

Trimberger, Ellen. 1977. Revolution From Above: Military Bureaucrats and Development in J apan, Turkey, Egypt and Peru. New Brunswick, NJ : Transaction Books.

Touraine, Alain. 1977. The Self-Production of Society (1973). Chicago: The University of Chicago Press.

Tuesta, Fernando. 2004. Una introducción a la ciencia política. Documento inédito.

Tuesta, Fernando. 1995. Sistemas de partidos políticos en el Perú, 1978-1995. Lima: Fundación Friedrich Ebert.

Tuesta, Fernando (ed.). 1996. Los enigmas del poder, Fujimori 1990-1996. Lima: Fundación Friedrich Ebert.

Tuesta, Fernando (ed.). 1999. El juego político. Fujimori, la oposición y las reglas. Lima: Fundación Friedrich Ebert.

Webb, Richard. 1987. "La gestación del plan antinflacionario del Perú". El Trimestre Económico LIV (septiembre/número especial).

Webb, Richard. 1988. Stabilization and adjustment programmes and policies. Country study: Peru. Helsinski: WIDER (World Institute for Development Economics Research of the United Nations University).

Martín Tanaka es Doctor en Ciencia Política por la Facultad Latinoamericana de Ciencias Sociales (FLACSO), sede México. Actualmente es Director General del Instituto de Estudios Peruanos (IEP). Ha sido profesor del Departamento de Ciencias Sociales de la Pontificia Universidad Católica del Perú y profesor visitante en la Maestría de Ciencia Política de la Universidad de Los Andes en Bogotá. Recientemente ha sido Visiting Fellow en el Helen Kellogg Institute for International Studies de la Universidad de Notre Dame.

(E-mail:mtanaka@iep.org.pe) 